\title{
Performance Analysis of an Optical CDMA MAC Protocol With Variable-Size Sliding Window
}

\author{
Mohamed Aly A. Mohamed, Student Member, IEEE, Hossam M. H. Shalaby, Senior Member, IEEE, \\ and El-Sayed Abdel-Moety El-Badawy, Senior Member, IEEE
}

\begin{abstract}
A media access control protocol for optical codedivision multiple-access packet networks with variable length data traffic is proposed. This protocol exhibits a sliding window with variable size. A model for interference-level fluctuation and an accurate analysis for channel usage are presented. Both multipleaccess interference (MAI) and photodetector's shot noise are considered. Both chip-level and correlation receivers are adopted. The system performance is evaluated using a traditional average system throughput and average delay. Finally, in order to enhance the overall performance, error control codes (ECCs) are applied. The results indicate that the performance can be enhanced to reach its peak using the ECC with an optimum number of correctable errors. Furthermore, chip-level receivers are shown to give much higher performance than that of correlation receivers. Also, it has been shown that MAI is the main source of signal degradation.
\end{abstract}

Index Terms-Chip-level receiver, correlation receiver, MAC protocols, multiple-access interference (MAI), optical CDMA, shot-noise, sliding window protocol.

\section{INTRODUCTION}

$\mathbf{O}$ PTICAL FIBERS have been commonly used in many communications and computer networks due to its extremely high bandwidth and its very low power losses. To utilize this bandwidth, multiple-access techniques must be used. Examples are wavelength-division multiple-access (WDMA), time-division multiple-access (TDMA), and code-division multiple-access (CDMA) techniques. The CDMA technique has many advantages over WDMA and TDMA, such as system simplicity, unnecessary synchronization, and efficient use of available bandwidth.

In optical CDMA technique, a spreading sequence [such as an optical orthogonal code (OOC)] is assigned to each user. This sequence is used for self-addressing of packets; thus, the receiver can distinguish between the time overlapped packets received from different users. Multiple-access interference (MAI) due to nonperfect orthogonality among the assigned code is the main source of signal degradation. Both thermal and shot noises have minor effects on the system performance.

Many researchers have been engaged in studying the performance of different CDMA systems [11]-[23]. Most researchers have focused on the physical layer of the optical CDMA network. However, few studies have considered the media-access-

Manuscript received October 23, 2005; revised July 12, 2006.

M. A. A. Mohamed and H. M. H. Shalaby are with the Department of Electrical Engineering, University of Alexandria, Alexandria 21544, Egypt (e-mail: m_aly@ieee.org; shalaby@ieee.org).

E.-S. A-M. El-Badawy is with the Alexandria Higher Institute of Technology, Smouha, Alexandria 21311, Egypt (e-mail: sbadawy@ieee.org).

Digital Object Identifier 10.1109/JLT.2006.881839 control (MAC) sublayer protocols, which are the main objectives of this paper. An analysis of slotted optical CDMA systems is presented by Muchenheim and Hampicke [14]. In this system, time is slotted, and users are permitted to send packets at the beginning of a slot; thus, the interference level during a packet is constant. The effect of randomized time of arrival (TOA) [12], [13] is considered by Hsu and Li [15], where packets arrive at the receiver with different delays. The effect of randomized TOA appears in a packet capture probability, where the first received packet can be captured if there is a sufficient time delay before the second packet. An unslotted system was analyzed by Hsu and Li [17], where a frequency hop/time hop (FH/TH) CDMA system is proposed. Due to the presence of a frequency hop [18], the proposed system was characterized by low correlation coefficients among the error probabilities of successive bits. As a result, the bit error probabilities of different packet bits are considered as independent events; thus, the number of errors per packet can be approximated to a Poisson distribution. While in [19], So et al. have presented a model for unslotted system that considers the case of multiple packet messages in wireless CDMA networks.

Our aim in this paper is to analyze an unslotted optical CDMA packet network and measure its performance. Each terminal in the network breaks its variable-length message into a set of constant length packets. The message length is variable. Two main indicators of the system performance have been focused upon. The first is the network throughput in packets per packet duration, which tells the average number of successfully transmitted packets per packet duration. The second indicator is the network delay, which tells, on the average, how long, in terms of packet duration, the packet is successfully received. Upon a successful reception of packets, the receiver sends a positive acknowledgment to the transmitter. Packet failure, however, is detected due to lack of positive acknowledgement.

In WDMA networks, the available bandwidth is divided into wavelengths. The main problem is to assign a wavelength to only one user. Any overlapping between two or more packets at the same wavelength will destroy all of them [1]-[5]. However, in CDMA networks [6]-[23], the collided packets will not be totally destroyed; the signal degradation depends on the interference level and its duration. In our analysis, perfect packet capture method is assumed [11], [13]; this means that the receiver can distinguish between all received packets and capture all of them, but not all of them are successfully received. In this case, the width of the correlation function is so narrow that the receiver is able to differentiate between overlapped packets arriving with a delay equal to or greater than one bit. 


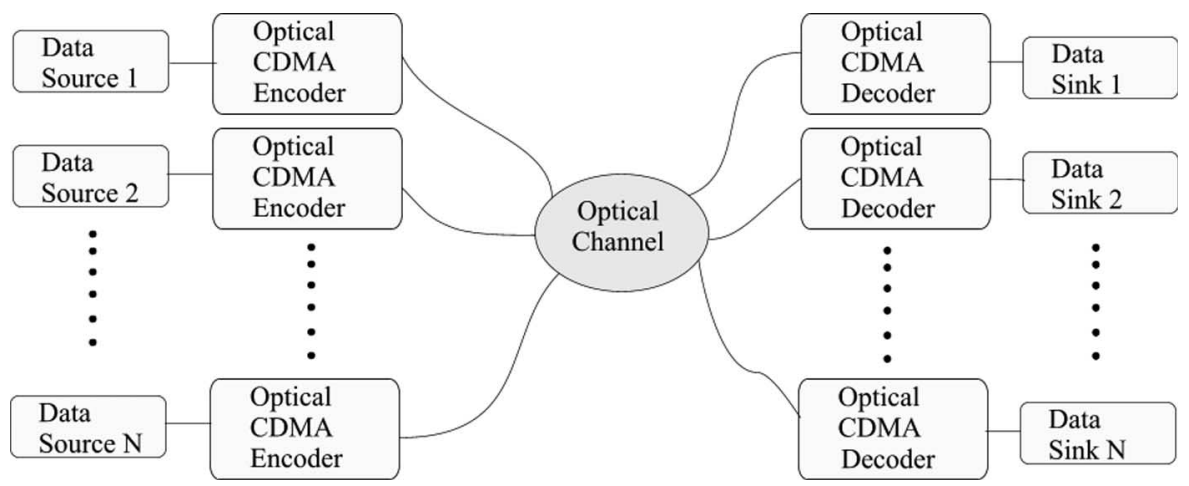

Fig. 1. Optical CDMA network.

The rest of this paper is arranged as follows. In Section II, the network architecture is presented. The mathematical model of the system is illustrated in Section III. In Section IV, the packet success probability, system throughput, and system delay are evaluated. Our numerical results are shown in Section V. Finally, this paper is concluded in Section VI.

\section{Network ARCHItecture}

The proposed network consists of a large number of users that can be considered as infinite population network. The network topology is a centralized network (star topology), i.e., a hub station is connected to all terminals (Fig. 1). Spread spectrum multiple-access technique is applied with a common spreading sequence, and the receiver can distinguish between time overlapped packets if there is a time offset that is greater than the width of the autocorrelation function of the used spreading sequence. At the physical layer, we consider both chip-level and correlation receivers.

The traffic offered to the system is assumed to be Poisson with an average rate of $\lambda$ messages/s. Each packet consists of a fixed number of bits ( $K$ bits/packet), and the message length $B$ (packets/message) is geometrically distributed with average length of $B_{\mathrm{av}}$ and a maximum length of $B_{\max }$; then, the message length in packets is $B \times K$. An error control code is applied and can correct up to $t$ errors/packet. The near-far effect is neglected, and all packets arrive to the receiver with equal power.

The system uses ON-OFF keying (OOK) modulation scheme and applies a spreading sequence from OOC family of $(N, w$, $\lambda_{\mathrm{a}}, \lambda_{\mathrm{c}}$ ), where $N$ is the code length, $w$ is the code weight, $\lambda_{\mathrm{a}}$ is the upper bound on the nonzero shift autocorrelation, and $\lambda_{\mathrm{c}}$ is the maximum values of cross-correlation function, respectively [6]-[8]. Both $\lambda_{\mathrm{a}}$ and $\lambda_{\mathrm{c}}$ are bounded to one, therefore, any interferer cannot interfere with more than one chip pulse.

It has been shown that the average bit error probability for the correlation receiver under the influence of MAI only is given by [7]

$$
P_{\mathrm{b}}(m)=\frac{1}{2} \cdot \sum_{i=w}^{m}\left(\begin{array}{c}
m \\
i
\end{array}\right) \cdot\left(\frac{w^{2}}{2 N}\right)^{i} \cdot\left(1-\frac{w^{2}}{2 N}\right)^{m-i}
$$

where $m$ is the number of interferers.
As for the chip-level receiver, the bit error probability with a shot noise and MAI is written as follows [9]:

$$
\begin{aligned}
P_{\mathrm{b}}(m)=\frac{1}{2} \cdot[1+ & \left.\sum_{i=1}^{w}(-1)^{i} \cdot\left(\begin{array}{c}
w \\
i
\end{array}\right)\right) \cdot\left(1-e^{-Q i}\right) \\
\cdot & {\left.\left[1-i \cdot \frac{w}{2 \cdot N}+i \cdot \frac{w}{2 \cdot N} \cdot e^{-Q}\right]^{m}\right] }
\end{aligned}
$$

where $Q$ is the average photons per a chip pulse. When $Q \rightarrow \infty$, the bit error probability reduces to [9]

$$
P_{\mathrm{b}}(m)=\frac{1}{2} \cdot\left[1+\sum_{i=1}^{w}(-1)^{i} \cdot\left(\begin{array}{c}
w \\
i
\end{array}\right) \cdot\left[1-i \cdot \frac{w}{2 \cdot N}\right]^{m}\right]
$$

\section{System AnAlysis AND MathematicAl Model}

In this section, we illustrate the mathematical model in order to evaluate both the system throughput and delay versus the system offered traffic. First, we evaluate the traffic offered to the system, then the transition of the interference level, and, finally, the packet success probability.

\section{A. Average Offered Traffic $(G)$}

The system average offered traffic is defined as the average number of generated packets in packet duration

$$
G=\lambda \cdot T_{p} \cdot B_{\mathrm{av}}
$$

where $T_{p}$ is the packet duration.

Since the length of a message is assumed to be geometrically distributed, the probability of a message to be of length $x$ is given by

$$
P_{B}(x)=\alpha \cdot p \cdot(1-p)^{x-1}
$$

where: $x \in\left\{1,2, \ldots, B_{\max }\right\}, p=1 / B_{\mathrm{av}}$, and

$$
\alpha=\frac{1}{1-(1-p)^{B_{\max }}} .
$$

In our system, the message length is limited to $B_{\max }$, and the factor $\alpha$ is a normalizing factor for the truncated geometric distribution. 


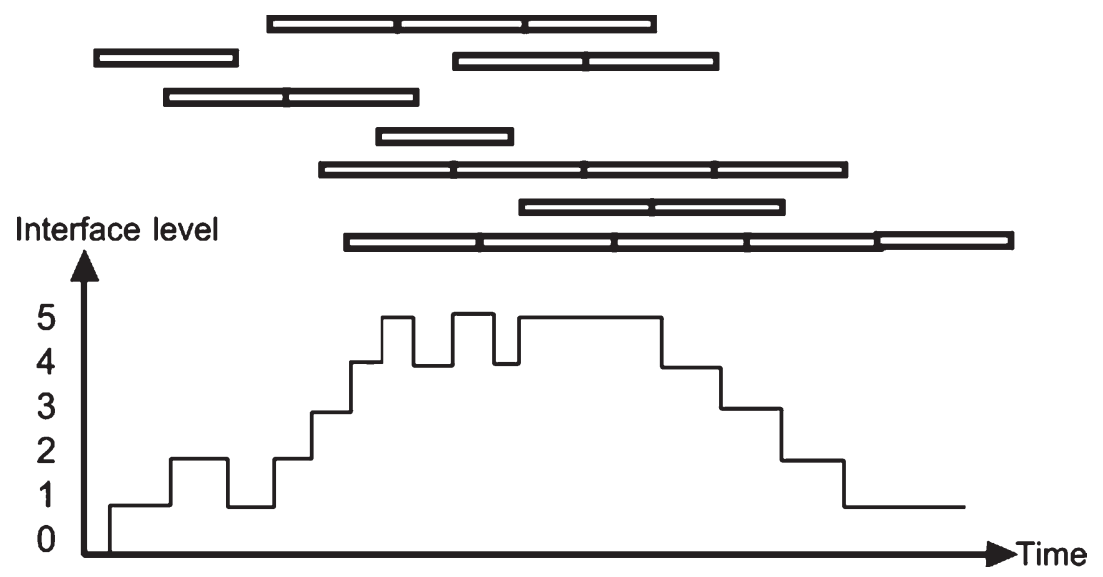

Fig. 2. Interference-level fluctuation.

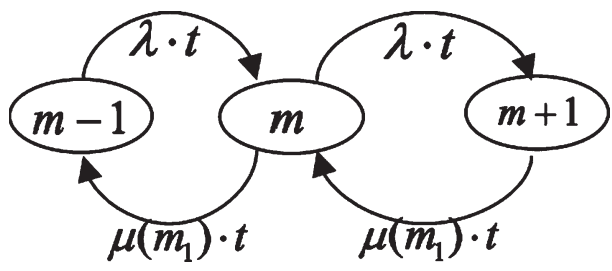

Fig. 3. Transition of the interference level.

\section{B. Modeling of the Interference Level and Transition}

The interference level of the unslotted systems is characterized by a continuous change (Fig. 2); we will study the interference level in each bit. We evaluate the probability of transition of the number of interfering messages as follows. Two sources of transition should be considered; the first is the generation of new messages, which is assumed to follow the Poisson distribution with arrival rate $(\lambda$ messages/s). The second is the termination of transmitted messages.

As for the generation of new messages, the probability of generating $k$ messages during $t \mathrm{~s}$ is given by

$$
P_{\mathrm{o}}(k, t)=\frac{(\lambda \cdot t)^{k}}{k !} \cdot e^{-\lambda t} .
$$

We found that for a data rate $R=256 \mathrm{~kb} / \mathrm{s}$, average message length $B_{\mathrm{av}}=2$, average offered traffic $G=5$ packets/packet duration, and a packet length $K=128$ bits, the probability of generating $k$-where $k=0,1,2,3,4,5$-messages in a bit duration is $\left\{0.98,0.019,1.8 \cdot 10^{-4}, 1.2 \cdot 10^{-6}, 5.9 \cdot 10^{-9}, 2.3\right.$. $\left.10^{-11}\right\}$. Thus, we will neglect the probability of generating more than one bit in a bit duration, and the transition of the interference level is limited to one.

As for the termination process, it is quite difficult to evaluate its rate, however, this process is also expected to follow the Poisson distribution. In the next section, we will evaluate the average death rate $\mu\left(m_{1}\right)$ in terms of the number of interferers at the beginning of the tagged packet $m_{1}$.

Next, we evaluate the interference level during the tagged packet. We assume a Markovian model in our analysis (Fig. 3).

Since the interference level can be changed by a value of one during a bit, and by assuming that the level is $m$ at a certain bit, the level of the next bit may be $m+1, m-1$, or $m$.

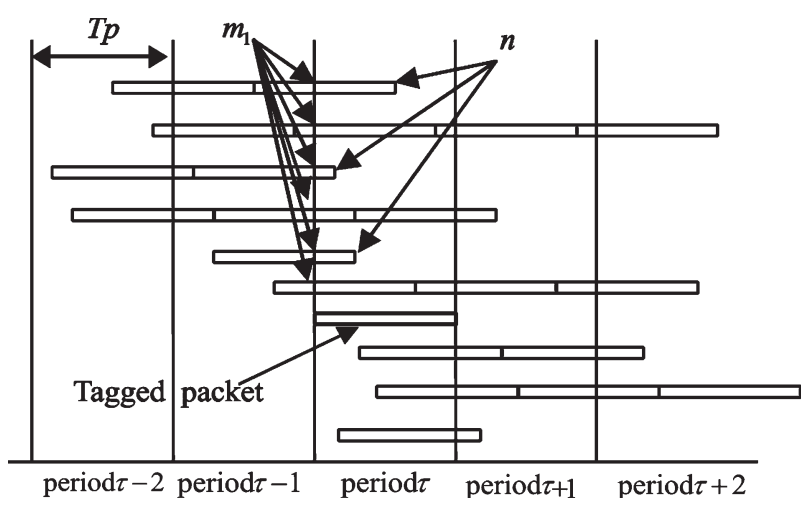

Fig. 4. Tagged packet analysis.

Thus, the transition probability can be calculated as follows:

$q\left(m_{j} \mid m_{j-1}\right)= \begin{cases}\lambda \cdot T_{\mathrm{b}}, & m_{j}=m_{j-1}+1 \\ \mu\left(m_{1}\right) \cdot T_{\mathrm{b}}, & m_{j}=m_{j-1}-1 \\ 1-\lambda \cdot T_{\mathrm{b}}-\mu\left(m_{1}\right) \cdot T_{\mathrm{b}}, & m_{j}=m_{j-1} \\ 0, & \text { otherwise }\end{cases}$

where $\mu\left(m_{1}\right)$ is the death rate, which is the rate of message's termination. Now, it is required to express the average death rate, this is to be discussed in the following steps.

As shown in Fig. 4, the tagged packet method is used to analyze the system. The time axis is divided into periods each of length $T_{P}$, the tagged packet occupies the period number $\tau$, and previous/next periods are $\tau-1, \tau-2 \ldots / \tau+1, \tau+$ $2 \ldots$ Let $\tau_{j}$ denote the $j$ th bit in the period $\tau$. In the tagged packet, $m_{j}$ is the interference level at $j$ th bit. The interference level during the tagged packet is changed continuously; for simplicity, it will be considered constant during each bit, and changes occur at the bit boundaries. In order to study the interference level during the tagged packet, we should consider both the generation and termination (death) rate. The generation rate is assumed to be a Poisson process. In this model, the level of initial interference $m_{1}$ is of great importance, where the death rate depends on how many interferers among $m_{1}$ will depart during the tagged packet.

It is now required to calculate $P_{\mathrm{I}}\left(m_{1}\right)$; which is the probability of initial interference level to be $m_{1}$. Define $a_{y}$ as the 
number of interferers existing at $\tau_{1}$ that have been generated in the period $\tau-y ; 1 \leq y \leq B_{\max }$, and $A=\left\{a_{1}, a_{2}, \ldots\right.$, $\left.a_{y}, \ldots\right\}$ is the set of all interferers generated in the previous $B_{\max }$ periods and exists at $\tau_{1}$.

To evaluate the probability of generating $a_{y}$ messages, consider that $k$ messages are generated in the period $\tau-y$, and any arbitrary $a_{y}$ messages among $k$ have a sufficient length to survive at the beginning of the tagged packet. This means that each of these messages has a length greater than or equal to $y$. Thus, $\operatorname{Pr}\left(a_{y}\right)$ follows the binomial distribution as follows:

$$
\begin{aligned}
& \operatorname{Pr}\left(a_{y}\right)=\sum_{k=a_{y}}^{\infty}\left(\begin{array}{c}
k \\
a_{y}
\end{array}\right) \cdot\left(P_{B}(B \geq y)^{a_{y}}\right. \\
& \cdot\left(1-P_{B}(B \geq y)^{k-a_{y}} \cdot P_{\mathrm{o}}\left(k, T_{P}\right)\right.
\end{aligned}
$$

where $P_{B}(B \geq y)=\sum_{B=y}^{B_{\max }} P_{B}(x)$.

Then, the probability to have a set of interferences $A$ : $\left\{a_{1}, a_{2}, \ldots a_{y} \ldots\right\}$ can be obtained by multiplying the probabilities of $a_{y}^{\prime} s$, as given by

$$
\operatorname{Pr}(A)=\prod_{y=1}^{B_{\max }} \operatorname{Pr}\left(a_{y}\right)
$$

Now, it is easy to calculate the probability of having $m$ initial interferences; it is the sum of the probabilities of all $A^{\prime}$ s whose summation is $m$ as

$$
P_{\mathrm{I}}(m)=\sum_{u} \operatorname{Pr}(A)
$$

where $u=\left\{A: \forall a_{y} \in A ; \sum_{y=1}^{B_{\max }} a_{y}=m\right\}$.

In this model, it is assumed that the packet termination is a Poisson process with an average rate of $\mu(m)$ packets/s. In order to evaluate the average death rate $\mu(m)$, suppose that the number of initial interferers in the tagged packet is equal to $m$, and that $n$ messages among $m$ will depart during the tagged packet. A message will depart during the tagged packet if it was initiated in the period $\tau-y$, and its length is equal to $y$. Define the probability $P_{\mathrm{d} 1}(m)$ as follows: the probability of one message to be of length $y$ and generated in the period $\tau-y$.

$$
P_{\mathrm{d} 1}\left(m_{1}\right)=\sum_{y=1}^{B_{\max }} \sum_{a_{y}=1}^{m_{1}} \operatorname{Pr}\left(a_{y}\right) \cdot a_{y} \cdot P_{x}(y) \cdot\left(1-P_{B}(y)\right)^{a_{y}-1} .
$$

Therefore, for a given value of initial interference $m_{1}$, the probability of $n$ messages to be terminated in the tagged period follows the binomial distribution as shown:

$$
\operatorname{Pr}\left(n \mid m_{1}\right)=\left(\begin{array}{c}
m_{1} \\
n
\end{array}\right) \cdot\left(P_{\mathrm{d} 1}\left(m_{1}\right)\right)^{n} \cdot\left(1-P_{\mathrm{d} 1}\left(m_{1}\right)\right)^{m_{1}-n}
$$

The average value of $n$ for a given value of $m$ is given by

$$
n_{\mathrm{av}}\left(m_{1}\right)=\sum_{n=1}^{m_{1}} n \cdot \operatorname{Pr}\left(n \mid m_{1}\right)
$$

Finally, we can express the average death rate for a given value of initial interference $m_{1}$ as follows:

$$
\mu\left(m_{1}\right)=\frac{n_{\mathrm{av}}\left(m_{1}\right)}{T_{P}} .
$$

\section{System Performance}

In this section, it is required to evaluate recursively the number of errors in the packet. The packet will be successfully transmitted if the number of errors is less than or equal to $t$, the number of correctable bits by an error control code (ECC). Define an error counter $f_{j}\left(e, m_{j}, m_{1}\right)$ as follows:

1) In the tagged packet, the first $j-1$ bits are transmitted with $e$ errors.

2) The number of interferers at the $j$ th bit is equal to $m_{j}$.

3) The number of initial interferers is equal to $m_{1}$.

This function is being used to calculate recursively the number of errors (error count) in the tagged packet.

At $j=1$, the first bit of the tagged packet $m_{j}=m_{1}$, and the error count should be equal to zero. This fact is used as an initial condition for the recursive calculations as follows:

$$
\begin{aligned}
& f_{1}\left(e=0, m_{1}, m_{1}\right)=P_{\mathrm{I}}\left(m_{1}\right) \\
& f_{1}\left(e>0, m_{1}, m_{1}\right)=0 .
\end{aligned}
$$

Now, it is required to evaluate the error count at the $j$ th bit of the tagged packet using the $f_{j}$ function. The function at the $j$ th bit depends on its value at the previous bit (the $j-1$ th bit). At the $j$ th bit, the error count is equal to $e$; this can occur in two cases.

1) The error count from the first bit until the $j-1$ th bit is equal to $e-1$, and an error occurred in the $j-1$ th bit.

2) The error count from the first bit until the $j-1$ th bit is equal to $e$, and the $j-1$ th bit is free of errors.

Considering the Markovian property of the interference-level transition, the $f_{j}$ function can be expressed as follows:

$$
\begin{aligned}
f_{j}\left(e, m_{j}, m_{1}\right)= & \sum_{m_{j-1}=m_{j}-1}^{m_{j}+1} f_{j-1}\left(e-1, m_{j}, m_{1}\right) \cdot q\left(m_{j} \mid m_{j-1}\right) \\
& \cdot P_{\mathrm{b}}\left(m_{j-1}\right)+\sum_{m_{j-1}=m_{j}-1}^{m_{j}+1} f_{j-1}\left(e, m_{j}, m_{1}\right) \\
& \cdot q\left(m_{j} \mid m_{j-1}\right) \cdot\left(1-P_{\mathrm{b}}\left(m_{j-1}\right)\right) .
\end{aligned}
$$

Finally, the packet success probability is the probability that the number of errors does not exceed $t$ which is the maximum correctable errors by Reed-Solomon (RS) codes, at the last bit of the tagged packet using the $f_{j}$ function until $j=K$ and 


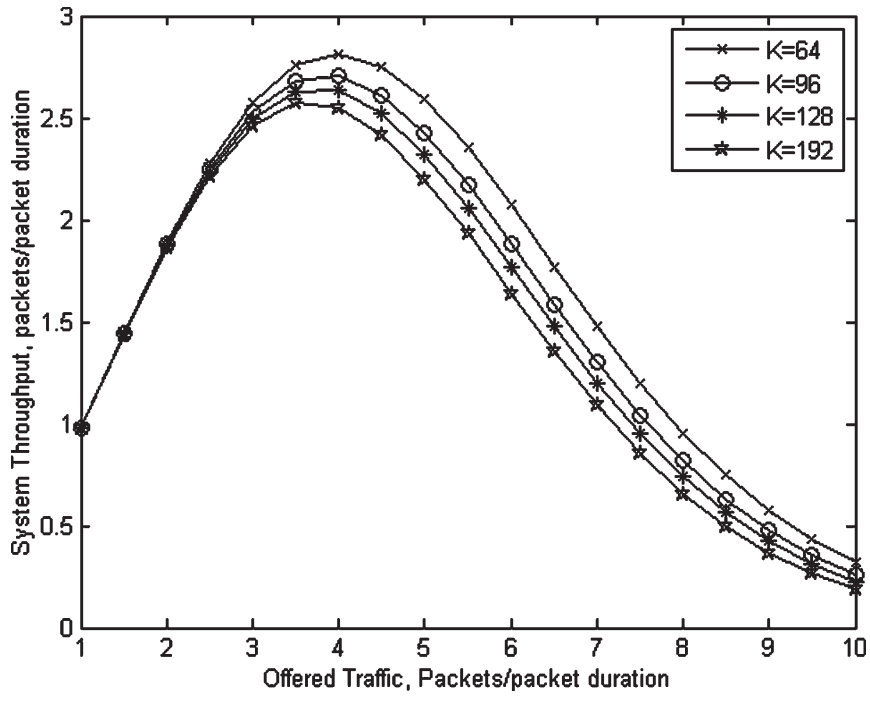

Fig. 5. System throughput versus offered traffic for the correlation receiver without ECC.

averaging over all possible values of $m_{K}$ and $m_{1}$. The packet success probability $Q_{s}(t)$ is expressed as follows:

$$
\begin{aligned}
Q_{s}(t)=\sum_{m_{K}=0}^{\infty} \sum_{m_{1}=0}^{\infty} f_{K}\left(t, m_{K}, m_{1}\right) \cdot\left(1-P_{\mathrm{b}}\left(m_{k}\right)\right) \\
+\sum_{e=0}^{t-1} f_{K}\left(e, m_{K}, m_{1}\right) .
\end{aligned}
$$

The last equation is based on the next two cases of the error count at the last bit of the tagged packet.

1) The error count at the last bit of the packet ( $K$ th bit) is equal to a value up to $t-1$, regardless of the error probability of the last bit.

2) The error count at the last bit is equal to $t$, and the ( $K$ th) bit is free of error.

The packet success probability $\left(Q_{s}\right)$ is given by (19), and the system throughput is given by

$$
S_{t}=G \cdot Q_{s}(t) \cdot \frac{s}{r}=G \cdot Q_{s}(t) \cdot\left(1-\frac{2 \cdot t}{K}\right) .
$$

The last factor in (20) considers the effect of bandwidth expansion by the RS coding, where $s$ is the number of data bits/code word of length $r$, and the number of correctable bits is given by $t=(r-s) / 2$. In this system, the packet is considered as one code word, i.e., $r=L$.

Finally, it is required to evaluate the average delay $D$, which can be expressed as the ratio between the system's offered traffic to the throughput. This can be expressed as follows:

$$
D_{t}=\frac{G}{S_{t}}
$$

\section{NUMERICAL EXAMPLES}

In this section, we will present some numerical results and examine the performance of the network in terms of the system throughput and average delay. The parameters used in the

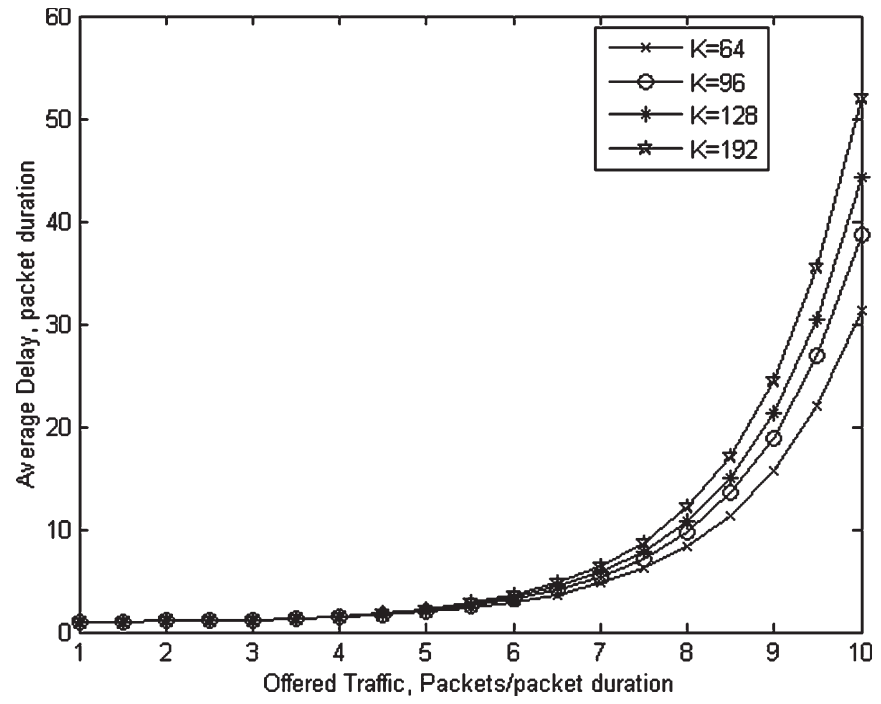

Fig. 6. Average delay versus offered traffic for the correlation receiver without ECC.

analysis are: bit rate of $256 \mathrm{~Kb} / \mathrm{s}$, average message length of two packets per message, and maximum message length of three packets per message. The used OOC is of length 127 and of weight 3.

We study the effect of varying the packet length (in bits) on the throughput and delay. Also, we apply the ECC with different correction capability. Although the use of the ECC is expected to raise the performance, we found that the increasing of the correction capability of the ECC (over optimum value) will reduce the throughput due to loosing more bandwidth.

Figs. 5 and 6 show the system throughput and delay versus the offered traffic for different values of $K$ (without ECC). We can see from Fig. 5 that as the packet length increases, the throughput is reduced, but another factor should be considered when choosing the packet length. That is, reducing the packet length, which requires more packets to send the message. Thus, the packet length should be optimized in order to obtain the maximum system performance.

Fig. 7 illustrates the enhancement in the system performance in both throughput and delay due to the use of ECC with different values of $t$. The use of ECC is expected to enhance the system performance. In fact, two factors should be considered when we study the effects of using the ECC. The first is the enhancement obtained in the packet success probability. This enhancement is proportional to the number of correctable bits. The second is the bandwidth expansion due to the addition of parity bits. Thus, the effective packet length (number of data bits per packet) is reduced, i.e., more packets are required to send the same message. As shown in Figs. 7 and 8, we can see that the optimum value of $t$ is 3 . This means that when increasing $t$ from 0 to 3 , the performance is enhanced; moreover, when $t$ exceeds the optimum value, the performance is reduced. This can be found clearly in Fig. 8, where we plotted the system throughput versus $t$ at $G=5$. From this figure, it is found that the throughput is enhanced and reached its maximum value at $t=3$; then, the curve will decay and the throughput is reduced.

Now, we present some results for systems with chip-level receivers. First, we evaluate the effect of ECCs; Fig. 9 shows 


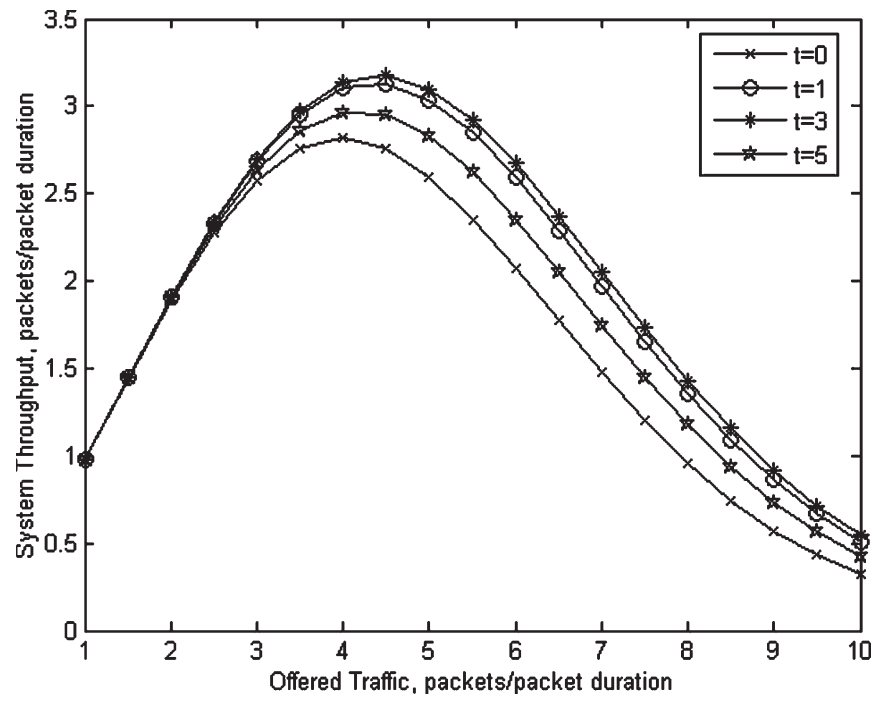

Fig. 7. System throughput versus offered traffic for the correlation receiver with different values of $t, K=64$.

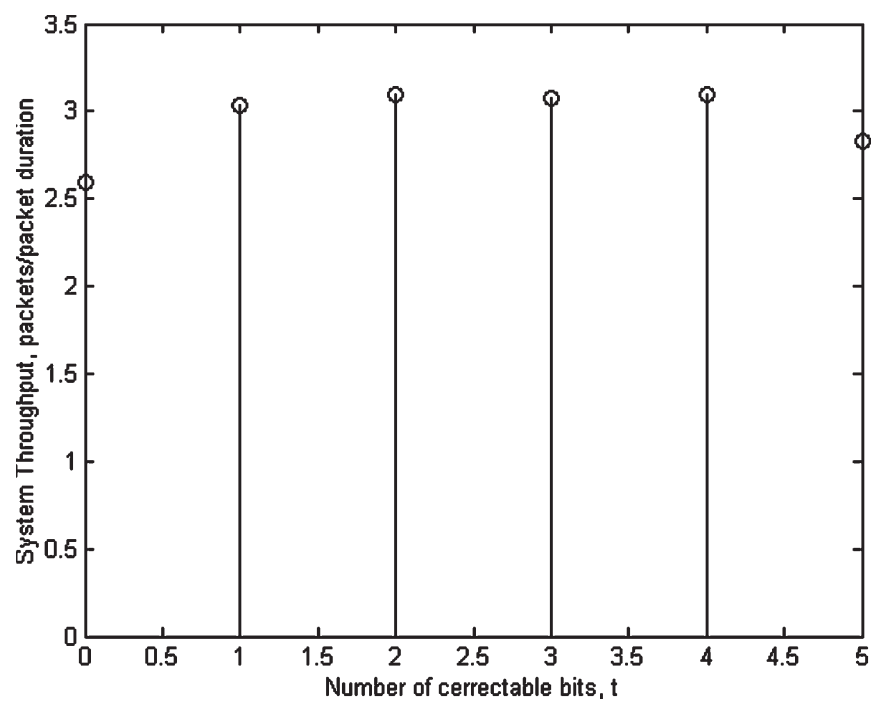

Fig. 8. System throughput versus number of correctable bits $t$ for the correlation receiver, $G=5, K=64$.

that similar to the correlation receivers, the system throughput increases as $t$ increases to reach a peak. When $t$ increases, moreover, the system throughput decreases.

Fig. 10 compares the performance of both correlation and chip-level receivers without ECCs for different values of packet length. It is clear that the system throughput achieved by the chip-level receiver is much higher than that achieved by the correlation receiver.

Finally, Fig. 11 presents the effect of the photodetector's shot noise on the performance of chip-level receiver. We have plotted the system throughput versus the average number of photons per chip pulse for different values of $t$ and a fixed packet length of 64 bits and offered traffic of five packets per packet duration. It is found that the system throughput reaches its maximum when $Q$ exceeds about ten photons per chip pulse. Hence, we can conclude that MAI is the major source of the signal degradation.

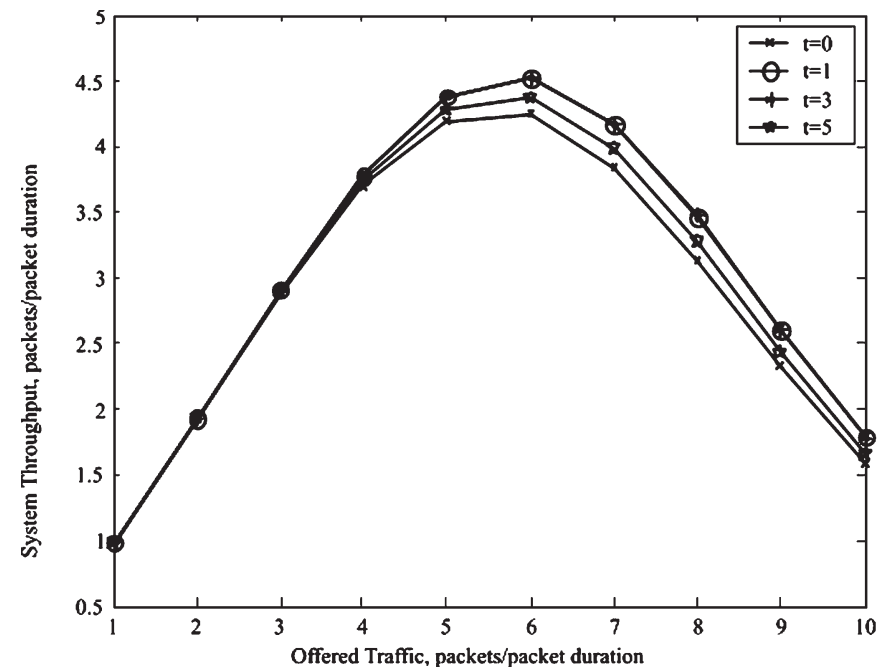

Fig. 9. System throughput versus offered traffic for chip-level receiver, $K=64$.

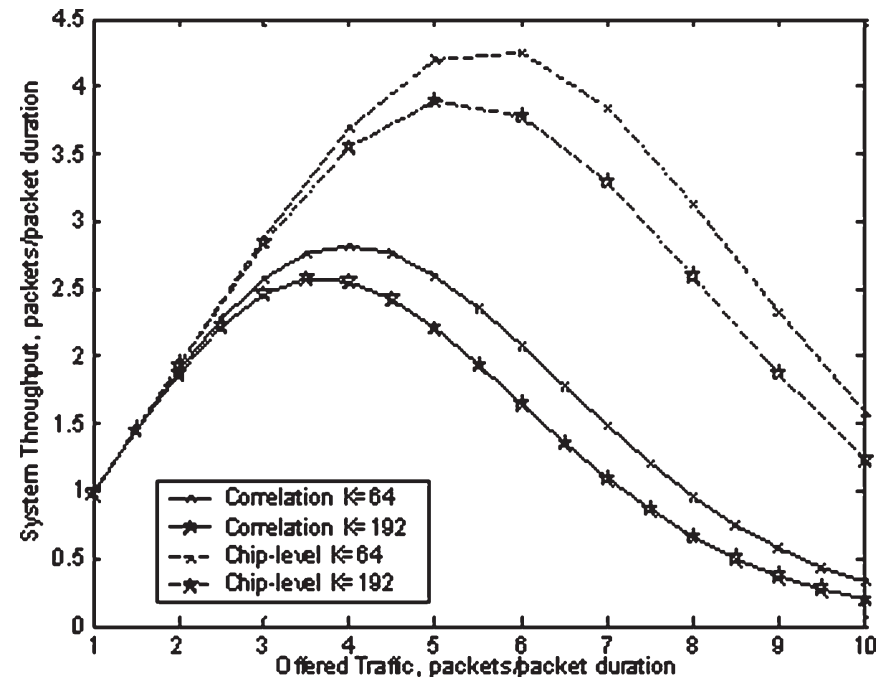

Fig. 10. System throughput versus offered traffic for both correlation and chip-level receivers.

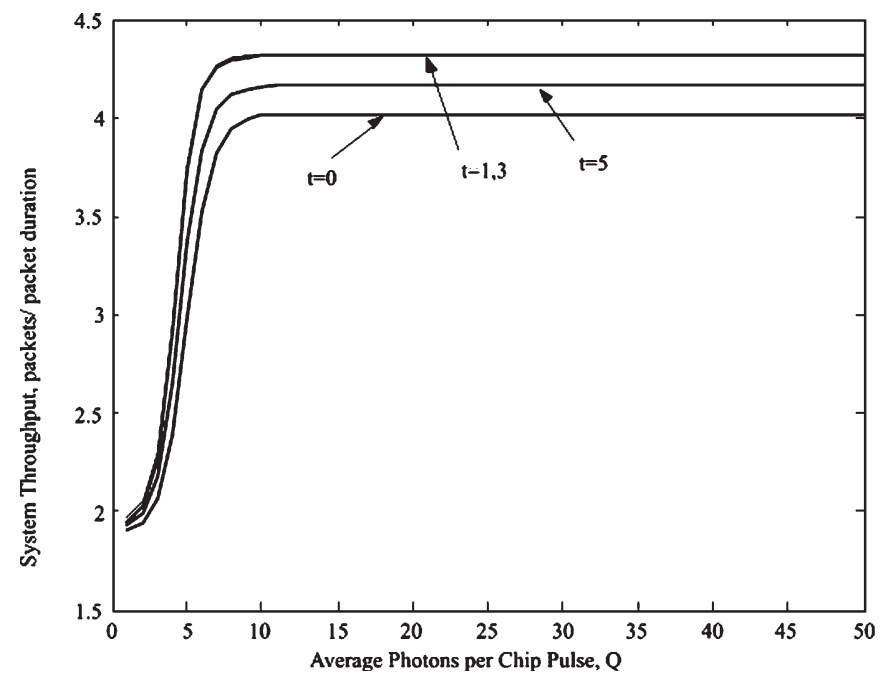

Fig. 11. System throughput versus the average number of photons per chip pulse for chip-level receiver, $G=5, K=64$. 


\section{CONCLUSION}

An accurate analysis of the MAC protocol used in optical CDMA networks is presented. The sliding window protocol with variable size is considered. The system throughput and average delay are evaluated, and an accurate description of the system state and channel usage is provided. Furthermore, the achieved enhancement by ECCs is examined. System performance is examined under the influence of both MAI and photodetector's shot noise. Results show the average system throughput and average delay versus the offered traffic and average number of photons per chip pulse as well as the effect of using ECC. Results indicate that system performance is enhanced with the increment of number of correctable bits $t$ reaching a maximum value. Moreover, as $t$ increases, the throughput decreases. It is also concluded that a significant improvement in performance can be achieved using the chiplevel receiver instead of the traditional correlation receiver. As for the effect of the photodetector's shot noise, we have found that it has a minor effect on the performance compared to MAI.

\section{REFERENCES}

[1] B. Mukherjee, "WDM-based local lightwave networks-Part I: Singlehop systems," IEEE Netw., vol. 6, no. 3, pp. 12-27, May 1992.

[2] I. M. I. Habbab, M. Kavehrad, and C. W. Sundberg, "Protocols for very high-speed optical fiber local area network using passive star topology," J. Lightw. Technol., vol. LT-5, no. 12, pp. 1782-1794, Dec. 1987.

[3] N. Mehravari, "Performance and protocol improvements for very high speed optical fiber local area networks using a passive star topology," J. Lightw. Technol., vol. 8, no. 4, pp. 520-530, Apr. 1990.

[4] S. S. Lam, "Packet broadcast networks-A performance analysis of the R-ALOHA protocol," IEEE Trans. Comput., vol. C-29, no. 7, pp. 596603, Jul. 1980.

[5] G. N. M. Sudhakar, N. D. Georganas, and M. Kavehard, "Slotted ALOHA and reservation ALOHA protocols for very high-speed optical fiber local area networks using passive star topology," J. Lightw. Technol., vol. 9, no. 10, pp. 1411-1422, Oct. 1991.

[6] J. A. Salehi, "Code division multiple-access techniques in optical fiber networks-Part I: Fundamental principles," IEEE Trans. Commun., vol. 37, no. 8, pp. 824-833, Aug. 1989.

[7] —-, "Code division multiple-access techniques in optical fiber networks-Part II: Systems performance analysis," IEEE Trans. Commun., vol. 37, no. 8, pp. 834-842, Aug. 1989.

[8] F. R. Chung, J. A. Salehi, and V. K. Wei, "Optical orthogonal codes: Design, analysis, and applications," IEEE Trans. Inf. Theory, vol. 35, no. 3, pp. 595-604, May 1989.

[9] H. M. H. Shalaby, "Chip-level detection in optical code-division multipleaccess," J. Lightw. Technol., vol. 16, no. 6, pp. 1077-1087, Jun. 1998.

[10] — " "Complexities, error probabilities, and capacities of optical OOK-CDMA communication systems," IEEE Trans. Commun., vol. 50, no. 12, pp. 2009-2017, Dec. 2002.

[11] N. Abramson, "Multiple access in wireless digital networks," Proc. IEEE, vol. 82, no. 9, pp. 1360-1370, Sep. 1994.

[12] D. H. Davis and S. A. Gronemeyer, "Performance of slotted ALOHA random access with delay capture and randomized time of arrival," IEEE Trans. Commun., vol. COM-28, no. 5, pp. 703-710, May 1980.

[13] M. G. Kyeong, "A novel algebraic framework for analyzing finite population DS/SS slotted ALOHA wireless network systems with delay capture," ERTU J., vol. 18, no. 3, pp. 127-145, Oct. 1996.

[14] J. Muchenheim and D. Hampicke, "Protocols for optical CDMA local area networks," in Proc. NOC, Antwerp, The Netherlands, 1997, vol. 1, pp. $255-262$.

[15] C.-S. Hsu and V. O. K. Li, "Performance analysis of slotted fiber-optic code-division multiple-access (CDMA) packet networks," IEEE Trans. Commun., vol. 45, no. 7, pp. 819-827, Aug. 1997.

[16] T. Sato, H. Okada, T. Yamazato, M. Katayama, and A. Ogawa, "Throughput analysis of DS/SSMA unslotted ALOHA system with fixed packet length," IEEE J. Sel. Areas Commun., vol. 14, no. 4, pp. 750-756, May 1996.

[17] C.-S. Hsu and V. O. K. Li, "Performance analysis of unslotted fiber-optic code-division multiple-access (CDMA) packet networks," IEEE Trans. Commun., vol. 45, no. 8, pp. 978-987, Aug. 1997.

[18] J. A. B. Tarr and J. E. Wieslthier, "Packet-error probability analysis for unslotted FH-CDMA systems with error control coding," IEEE Trans. Commun., vol. 38, no. 11, pp. 1987-1993, Nov. 1990.

[19] J. So, I. Han, B. Shin, and D. Cho, "Performance analysis of DS/SSMA unslotted ALOHA with variable length data traffic," IEEE J. Sel. Areas Commun., vol. 19, no. 11, pp. 2215-2224, Nov. 2001.

[20] H. M. H. Shalaby, "Optical CDMA random access protocols with and without pretransmission coordination," J. Lightw. Technol., vol. 21, no. 11, pp. 2455-2462, Nov. 2003.

[21] _ - "A proposal of an optical CDMA random access protocol," in Proc. IEEE ICC, Paris, France, Jun. 20-24, 2004, pp. 1709-1713.

[22] - "Performance analysis of an optical CDMA random access protocol," J. Lightw. Technol., vol. 22, no. 5, pp. 1233-1241, May 2004.

[23] M. A. A. Mohamed, H. M. H. Shalaby, and E.-S. A. El-Badawy, "Variable-size sliding window optical CDMA MAC protocol," in Proc. 46th IEEE Int. MWSCAS, Cairo, Egypt, Dec. 27-30, 2003, pp. 496-500.

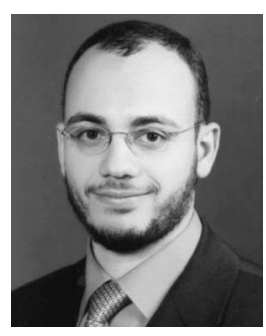

Mohamed Aly A. Mohamed (S'99) was born in Alexandria, Egypt, in 1979. He received the B.Sc. and M.Sc. degrees in electrical engineering from the Department of Electrical Engineering, Faculty of Engineering, University of Alexandria, in 2001 and 2004, respectively, where he is currently working toward the Ph.D. degree in the field of mobile CDMA communications systems.

He started his academic career as a Teaching Assistant with the University of Alexandria in 2001 and for two years before joining Thebes Higher Institute of Engineering, Thebes Academy, Cairo, Egypt, first as a Teaching Assistant and then as a Lecturer Assistant for about two years. Since September 2004, he has been with Huawei Technologies Company, Cairo. Huawei is one of the leading worldwide companies working in the communications area. He is working as a Product Manager in application and software department. He is the Head of analyzing and designing solutions for value added services (VAS) covering different communication networks from PSTN, NGN, and mobile networks $(2 \mathrm{G}, 2.5 \mathrm{G}$, and $3 \mathrm{G})$. His current research interests are in the areas of CDMA, optical and wireless communications, and network protocols, and he has published several papers in these areas.

Mr. Mohamed received the 2005 Award of Best Individual Performance of Application and Software Development, Huawei Technologies Company. 


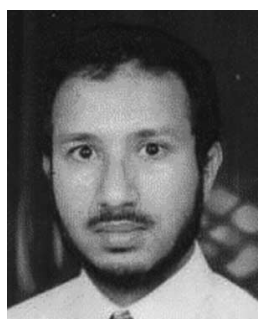

Hossam M. H. Shalaby (S'84-M'91-SM'99) was born in Giza, Egypt, in 1961. He received the B.S. and M.S. degrees from University of Alexandria, Alexandria, Egypt, in 1983 and 1986, respectively, and the Ph.D. degree from University of Maryland, College Park, in 1991, all in electrical engineering.

In 1991, he joined the Department of Electrical Engineering, University of Alexandria, as an Assistant Professor. He was promoted to the position of an Associate Professor in 1996 and then to Professor in 2001 (current position). From December 2000 to 2004, he was an Adjunct Professor with the Department of Electrical and Information Engineering, Faculty of Sciences and Engineering, Laval University, Quebec City, QC, Canada. From March to April 1996, he was a Visiting Professor with the Electrical Engineering Department, Beirut Arab University, Beirut, Lebanon. From September 1996 to January 1998, he was an Associate Professor with the Electrical and Computer Engineering Department, International Islamic University Malaysia (IIUM), Kuala Lumpur, and from February to December 1998, he was with the School of Electrical and Electronic Engineering, Nanyang Technological University, Singapore, where he was a Senior Lecturer and, from January 1999 to February 2001, an Associate Professor. His research interests include optical communications, optical CDMA, spread-spectrum communications, and information theory.

Dr. Shalaby received the SRC fellowship from 1987 to 1991 (Systems Research Center, MD), the Shoman Prize for Young Arab Researchers in 2002 (The Abdul Hameed Shoman Foundation, Amman, Jordan), the State Award twice in 1995 and 2001 (Academy of Scientific Research and Technology, Egypt), the University Award in 1996 (University of Alexandria), and the Soliman Abd-El-Hay Award in 1995 (Academy of Scientific Research and Technology, Egypt). He has served as a student branch counselor (University of Alexandria), IEEE Alexandria and North Delta Subsection from 2002 to 2006, and served as a chairman of the Student Activities Committee of the IEEE Alexandria Subsection from 1995 to 1996.

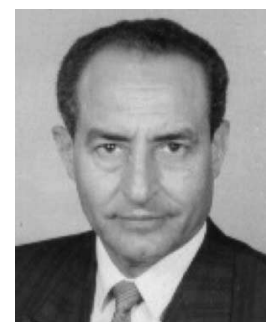

El-Sayed Abdel-Moety El-Badawy (SM'96) was born in Damietta, Egypt, on October 24, 1941. He received the B.Sc. degree (with distinction) in electrical engineering from the University of Alexandria, Alexandria, Egypt, in 1964 and the D.E.A D'Electronique and the Ph.D. degree (Docteur Ingénieur) with first degree of honor and written congratulations of the jury from the Université Paris VI, Paris, France, in 1971, and 1974, respectively.

In 1964, he joined the Department of Electrical Engineering, Faculty of Engineering, University of Alexandria, as a Teaching Assistant and was then promoted to Assistant Professor in 1974, Associate Professor in 1979, Professor of communications and electronics in 1984, and Senior Professor in 1994. He was seconded as Professor of communications and electronics with the Beirut Arab University, Beirut, Lebanon (1983-1987), the King Saudi University, Riyadh, Saudi Arabia (1991-1995), and the International Islamic University Malaysia (IIUM), Kuala Lumpur, Malaysia (1995-2000). He worked as a Visiting Professor (on short-term basis) with the University of California, Los Angeles, and the Université Paris VI (1980 and 1982), as well as a Visiting Professor (on parttime basis) at many Egyptian universities, institutions, and advanced technical schools. From April 2003 to December 2005, he was the Dean of the Thebes Higher Institute of Engineering, Thebes Academy, El-Maadi, Cairo, Egypt. $\mathrm{He}$ is currently the Dean of the Alexandria Higher Institute of Technology (AIT), Smouha, Alexandria, and an Emeritus Professor with the Department of Electrical Engineering, Faculty of Engineering, University of Alexandria. $\mathrm{He}$ has a wide experience in teaching, research, consultation, administration, and other extracurricular activities. He has supervised $15 \mathrm{Ph} . \mathrm{D}$. and $30 \mathrm{M}$.Sc. theses and published about 172 research papers in the fields of lasers (construction, propagation, modulation, and applications), electronics and optoelectronics, communications and optical fiber communications, and microwave devices and circuits. He has participated in more than 115 conferences where he presented papers. He has authored or coauthored many books and monographs in many areas of electrical engineering and advanced engineering mathematics. He has reviewed and refereed many books, projects, and papers for many scientific local, regional, and international journals and conferences.

Prof. El-Badawy has been a member of the Optical Society of America since 1980. He has been a member of many technical, scientific, and international advisory committees, the Chairman and Co-Chairman of many technical sessions, and the General Chairman of one conference. He was the Founder and the Chief Editor of the IIUM Engineering Journal from October 1998 to 2000. 